\title{
Temperature Calculations of Heat Loads in Rotating Target Wheels Exposed to High Beam Currents*
}

\author{
John P. Greene, Rachel Gabor ${ }^{\dagger}$ and Janelle Neubauer ${ }^{\ddagger}$ \\ Physics Division, Argonne National Laboratory, 9700 S. Cass Avenue, Argonne, IL 60439 USA
}

\begin{abstract}
In heavy-ion physics, high beam currents can eventually melt or destroy the target. Tightly focused beams on stationary targets of modest melting point will exhibit short lifetimes. Defocused or "wobbled" beams are employed to enhance target survival. Rotating targets using large diameter wheels can help overcome target melting and allow for higher beam currents to be used in experiments. The purpose of the calculations in this work is to try and predict the safe maximum beam currents which produce heat loads below the melting point of the target material.
\end{abstract}

\section{Introduction and Motivation}

In an effort to predict the lifetimes of targets under heavy-ion bombardment due to melting of the target material, calculations have been performed of the heat dissipation occurring within the target. For our research involving the production of heavy elements, the reaction cross-sections are small. Many days of beam time at high beam currents $(Z 10 \mathrm{pnA})$ on target are encountered. The targets produced for these experiments must be robust and capable of withstanding the intense currents involved. Calculations of the heating within the target can give a guideline to the safe limits to which they may be exposed without melting. However, melting is not the only factor involved in target longevity. Sputtering, stress due to radiation damage, recrystallization, changes in emissivity and plastic deformation all can play destructive roles. In this work we will concentrate on the more straight forward target failure due to melting, and attempt to predict when this mode of failure occurs.

\section{Description of the Calculations}

In heavy-ion physics, high beam currents will eventually destroy targets through melting, sputtering or radiation damage. Tightly focused beams impinging on stationary targets of modest melting point material lead to short target lifetimes as beam

*Work supported by the U.S. Department of Energy, Nuclear Physics Division. Contract No. W-31-109-ENG-38.

"Harvey Mudd College, Claremont, California

${ }^{\ddagger}$ North Central College, Naperville, Illinois currents are raised. Defocused or "wobbled" beams enhance target survival. However, rotating target wheels can often overcome target melting and allow for high beam currents to be used in experiments. The calculations in this work are aimed at predicting the safe range of beam currents which produce heat loads below the melting point of the target material. For our research involving reactions with small cross-sections, many days of experimental beam time using high beam currents are normal. The targets produced for these experiments must be robust and capable of withstanding the beam currents involved. Calculations of the heating within these targets give a guideline to the safe maximum beam currents they may be exposed to before melting.

The calculation must take into account how much heat the beam produces in the target, and how that heat is dissipated as the target rotates. The balanced equation of "heat in" = "heat out" needs to be satisfied. Heat is brought into the target by the energy loss of the beam inside the foil. This is known as the energy loss and may be calculated using a stopping power model such as TRIM [1]. Under fixed conditions, the heat produced is proportional to the beam current. The heat in the target decreases over time by conduction through the foil away from the beam spot given by the equation:

$$
Q=\left(T-T_{0}\right) \lambda A / d
$$

where $\lambda$ is the thermal conductivity, $\mathrm{A}$ is the crosssectional area and $d$ the distance through the foil away from beam spot. In addition the target heating is dissipated by radiation as given by: 


\section{DISCLAIMER}

This report was prepared as an account of work sponsored by an agency of the United States Government. Neither the United States Government nor any agency thereof, nor any of their employees, make any warranty, express or implied, or assumes any legal liability or responsibility for the accuracy, completeness, or usefulness of any information, apparatus, product, or process disclosed, or represents that its use would not infringe privately owned rights. Reference herein to any specific commercial product, process, or service by trade name, trademark, manufacturer, or otherwise does not necessarily constitute or imply its endorsement, recommendation, or favoring by the United States Government or any agency thereof. The views and opinions of authors expressed herein do not necessarily state or reflect those of the United States Government or any agency thereof. 


\section{DISCLAIMER}

Portions of this document may be illegible in electronic image products. Images are produced from the best available original document. 


$$
E=\varepsilon \sigma S\left(T^{4} T_{0}{ }^{4}\right)
$$

where $\sigma$ is the Stephan-Boltzman constant, $S$ is the surface area irradiated by the gaussian shaped beam and $\varepsilon$ is the emissivity of the material. A FORTRAN program [2] was developed to calculate the temperature distribution for the target material in the form of a wheel. To simulate wheel rotation, the power produced by the beam is applied to a slab of target material over a short time interval (beam on) and then removed. The target slab is then left to cool (beam off) until it again rotates into the beam. The basic equation is given as follows:

$$
W I=m C_{v} d T / d t+\left(T-T_{0}\right) \lambda D / \rho+2 \varepsilon \sigma S\left(T^{4}-T_{0}^{4}\right)
$$

with $W$ equal to the energy loss, I the beam intensity, $m$ is the amount of mass in the beam and $C_{v}$ is the specific heat. In the next term, $D$ is the target thickness (mass per unit area) and $\rho$ is the density of the target material. In the last term, the factor 2 takes into account that energy is radiated from both sides of the foil. The resulting time-dependent partial differential equation for temperature was solved using the finite difference method [3].

The program code was then executed for our beam/target systems and the maximum target temperature was calculated as a function of beam current. The results clearly show the maximum beam current that keeps the target temperature below the melting point. In addition, the time dependence of the temperature within a slab of target material following the wheel rotation (beam on/beam off) was studied. Figure 1 shows plots of the target temperature vs. time for a $449 \mathrm{MeV}, 2$ pnA ${ }^{86} \mathrm{Kr}$ beam irradiating $0.5 \mathrm{mg} / \mathrm{cm}^{2} \mathrm{~Pb}$ and $\mathrm{Bi}$ target wheels rotating at $600 \mathrm{RPM}$. The contrasting behavior of their temperature dependence may be attributed to differences in the emissivity-and thermal conductivity of these two metals.

\section{Input Parameters}

The program requires as input the radius and speed of the rotating target. For the target material, several thermal properties are needed. The specific heat, $C_{v}$, gives a measure of the energy required to raise the temperature within the target, while the values for thermal conductivity and emissivity provide the means of lowering the target temperature. Table 1 gives the input parameters used for the calculations. The value for the emissivity of
$\mathrm{PbS}$ was estimated to be 0.3 based on the spectral emissivity [4] and from similar values for $\mathrm{PbO}$.

For the beam, the energy, current and beam spot size are needed as input. The energy loss was calculated using the thickness and material comprising the target. The power per unit area deposited in the target was determined using the energy loss and beam current but was also highly dependent on the size of the beam spot on the target.
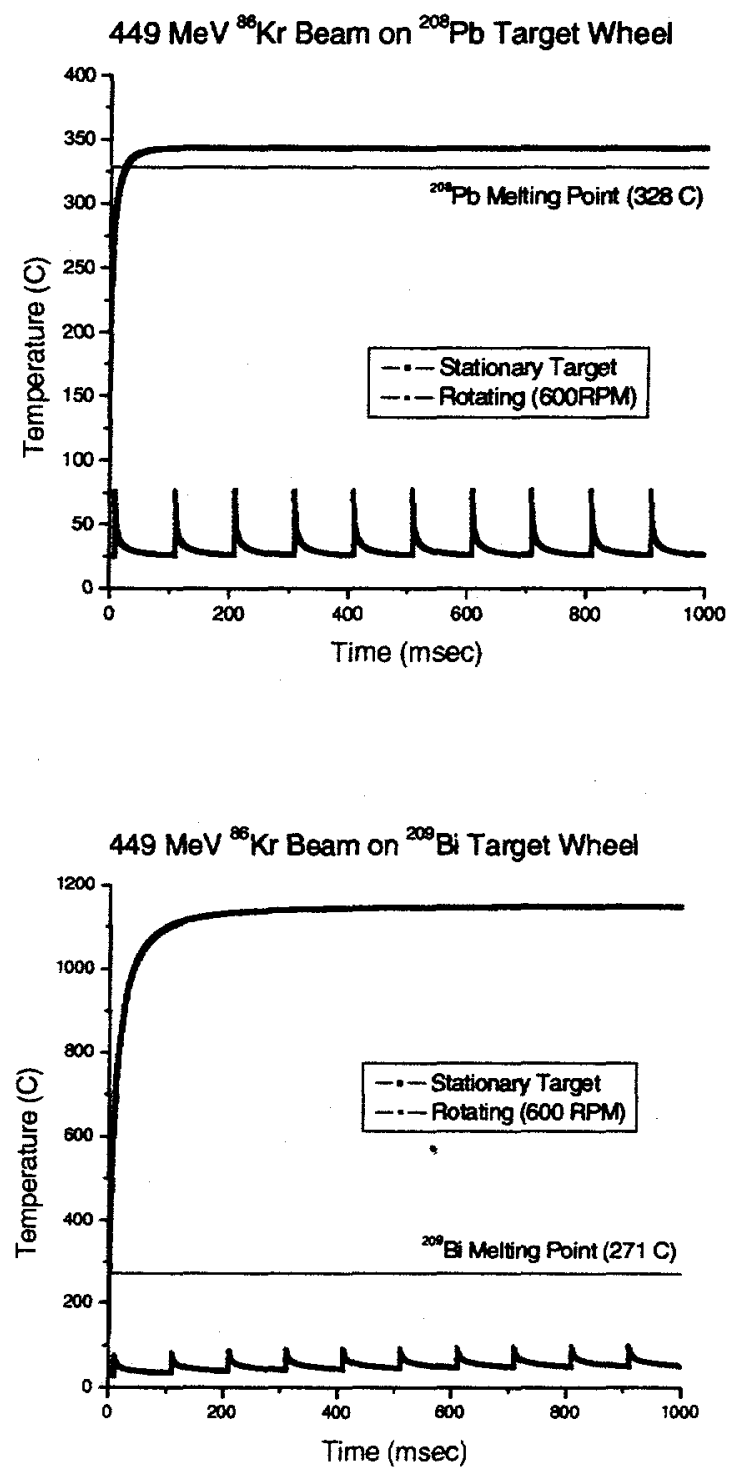

Figure 1. Plots of the target temperature vs. time for a $449 \mathrm{MeV}{ }^{86} \mathrm{Kr}$ beam of current $2 \mathrm{pnA}$ irradiating 0.5 $\mathrm{mg} / \mathrm{cm}^{2} \mathrm{~Pb}$ and $\mathrm{Bi}$ target wheels rotating at $600 \mathrm{RPM}$.

RECEIVED

JAN 052001 
Table 1.

\begin{tabular}{|l|c|c|c|c|c|c|}
\hline Target & Emissivity [6] & $\begin{array}{c}\text { Specific Heat } \\
(\mathbf{J} / \mathbf{g}-\mathbf{K})[7]\end{array}$ & $\begin{array}{c}\text { Density } \\
\left(\mathbf{g} / \mathbf{c m}^{3}\right)\end{array}$ & $\begin{array}{c}\text { Thermal Conductivity } \\
(\mathbf{W} / \mathbf{m}-\mathbf{K})[7]\end{array}$ & $\begin{array}{c}\text { Energy Loss for } \\
449 \mathbf{M e V}^{\mathbf{8}} \mathbf{K r}\end{array}$ & $\begin{array}{c}\text { Melting } \\
\text { Point }\left({ }^{\circ} \mathbf{C}\right)\end{array}$ \\
\hline $\mathbf{P b}$ & 0.43 & 0.1288672 & 11.35 & 34.4 & 17.7 & 328 \\
\hline $\mathbf{B i}$ & $0.048[7]$ & 0.1221728 & 9.74 & 7.22 & 18.0 & 271 \\
\hline $\mathbf{P b O}$ & 0.28 & 0.2050760 & 9.53 & 2.77 & 19.2 & 886 \\
\hline $\mathbf{P b S}$ & 0.3 (est.) & 0.2068396 & 7.50 & 2.30 & 19.5 & 1114 \\
\hline
\end{tabular}

The program assumes a Gaussian shape to the beam with the highest current density contained in the peak of the distribution. From our previous example, for a $449 \mathrm{MeV}{ }^{86} \mathrm{Kr}$ beam with a current of $2 \mathrm{pnA}$ and a focused spot size of $0.5 \mathrm{~mm}$, the peak power deposited in a $0.5 \mathrm{mg} / \mathrm{cm}^{2}{ }^{208} \mathrm{~Pb}$ stationary target was calculated to be 2.3 watts $/ \mathrm{cm}^{2}$. This translates to a maximum temperature within the target of $343^{\circ} \mathrm{C}$, which is reached rather quickly $(-50 \mathrm{msec})$. For a high current, tightly focused beam, the energy deposited would quickly vaporize the target material, and so the beam may be intentionally defocused. This may produce unwanted effects in the experiment.

A more practical approach used in many laboratories is to rotate the target. Using rotation, the small target section heated for a brief time interval by the beam spot, is allowed to cool until it once again rotates into the beam. The radius of the wheel and its speed of rotation determine this heating/cooling cycle. The limitations then become the preparation of large, segmented, target wheels and the availability and performance of motor drives and linkages for high speed rotation. In Figure 2, these effects are shown in a plot of maximum target temperature vs. beam current for the $449 \mathrm{MeV}{ }^{86} \mathrm{Kr}$ beam on ${ }^{208} \mathrm{~Pb}$ targets. For the target wheel $(r=22$ $\mathrm{mm}$ ) rotating at $600 \mathrm{RPM}$, the melting point for the lead target (m.p. $328^{\circ} \mathrm{C}$ ) is reached at a calculated beam current of about 12 pnA.

A further method employed involves "wobbling" the beam using a triangular waveform at approximately $5 \mathrm{~Hz}$, using magnetic steerers upstream from the target position. The "wobbling" produces an amplitude about the vertical dimension, effectively increasing the area the beam illuminates on the target as it rotates. In essence the focused beam is traveling with a higher velocity (rotation plus "wobbling") across the target foil. Moreover, because of these non-coupled motions, the beam does not return to the same point on the target after one complete rotation of the wheel. This allows even further cooling to occur. Further work is needed to refine the calculation to include these effects.

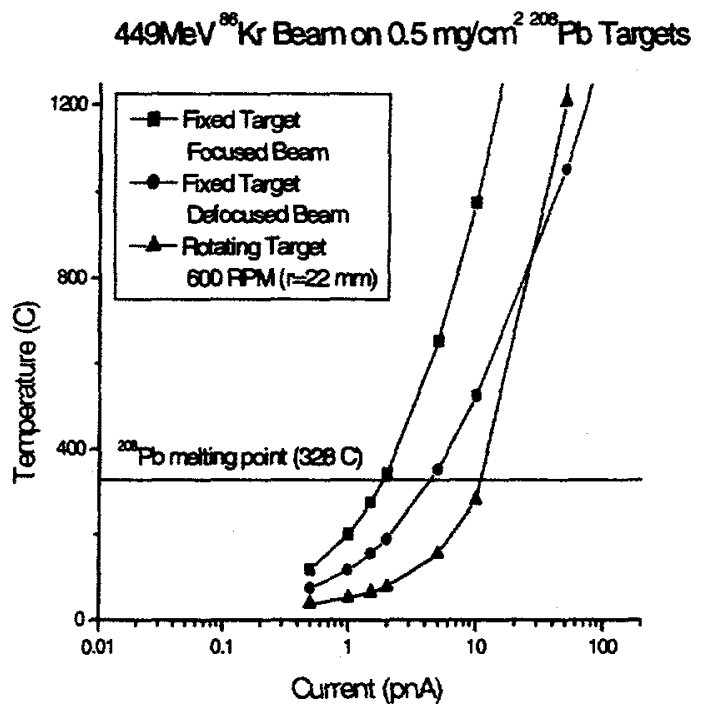

Figure 2. Plot of Temperature vs. Beam Current for an ${ }^{86} \mathrm{Kr}$ beam on $\mathrm{a}^{208} \mathrm{~Pb}$ target wheel showing the effect of the beam dimensions and rotation.

\section{Results of the Calculations}

Calculations were carried out which explored the effects of rotation speed and wheel radius on target heating. For rotation speed, increasing the speed allows higher beam currents to be used before target melting. One can observe the rise and fall in temperature following a point on the target as it encounters the beam and then rotates away. Higher speed decreases this amplitude, shortening the cooling period before the next beam encounter. Ulimately we are constrained by the maximum speed of the motor drive systems employed $(-1000$ RPM). The temperature rise within the target as a 
function of wheel radius was investigated for two cases; small $(r=22 \mathrm{~mm})$ and large $(r=88 \mathrm{~mm})$ wheels already in use for experiments. By rotating the target and employing beam wobbling, using the beam parameters as before, the power deposited in the $\mathrm{Pb}$ target is reduced to $2.6 \mathrm{~mW} / \mathrm{cm}^{2}$ for the small wheel and $0.64 \mathrm{~mW} / \mathrm{cm}^{2}$ for the large wheel as compared to a stationary target. The large target wheel ( $r=88$ mm) was designed to operate at 1000 RPM. This allows an order of magnitude increase in beam current before the onset of target melting.

Calculations were then performed for several target systems currently under study. A beam of 449 $\mathrm{MeV}^{86} \mathrm{Kr}$ was used to bombard four targets; $\mathrm{Pb}, \mathrm{Bi}$, $\mathrm{PbO}$ and $\mathrm{PbS}$, and employing the GAMMASPHERE target wheel $(\mathrm{r}=22 \mathrm{~mm})$ [5]. The results, plotted in Figure 3 show the limits of beam current the targets are calculated to withstand with respect to their melting points. In practice, target damage occurs before this limit of target melting is reached. For example, in $\mathrm{Pb}$ targets, island formation and migration of the lead material away from the beam heating is observed, leading to limited useful lifetimes.

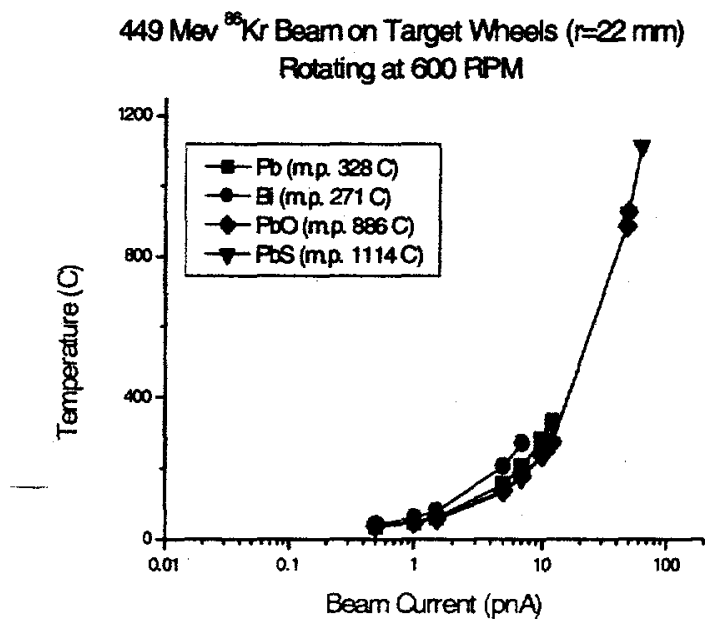

Figure 3. Plot of Temperature vs. Beam Current for $\mathrm{Pb}$, $\mathrm{Bi}, \mathrm{PbO}$ and $\mathrm{PbS}$ target wheels.

\section{Conclusion and Future Considerations}

In conclusion, the calculations performed showed the variation in target heating due to rotation speed and radius of the target wheel. The calculations also dramatically show the sensitivity of the target heating to the beam shape, which is why the beam is wobbled in addition to the target rotation. Preliminary experimental results are supported by the calculations. The initial tests, although intended primarily to explore accelerator capabilities and detector performance, irradiated targets at beam currents up to $15 \mathrm{pnA}$. The $\mathrm{Pb}$ and $\mathrm{Bi}$ targets were able to survive at this current using beam wobbling. The less robust $\mathrm{PbO}$ and $\mathrm{PbS}$ targets showed signs of rupture, although it is not clear that the damage was beam induced. Higher beam currents are expected in proposed heavy element synthesis experiments and calculations of this type will prove valuable in predicting target behavior under actual conditions. Further modifications to the calculations will include the contribution from the carbon backing foils used and for gas cooling of the target wheel.

\section{Acknowledgments}

The authors would like to acknowledge the previous research of Dr. Birger Back and James P. Done, a summer student working for him, upon which the present work is based. The experimental work was carried out under the direction of Dr. Robert Janssens.

We would also like to thank Dr. Donald Geesaman, the Physics Division Director, and Dr. Irshad Ahmad, the Target Facility Group Leader, for their continuing encouragement and support of these efforts. This work is supported by the U.S. Department of Energy, Nuclear Physics Division, under Contract No.W-31-109-Eng-38.

\section{References}

[1] J.F. Ziegler, J.P. Biersack and U. Littmark, The Stopping and Range of Ions in Solids, Pergamon Press, New York, USA 1985.

[2] B. Back, priv. comm.

[3] W.H. Press, S.A. Teukolski, W.T. Vetterling and B.P. Flannery, Numerical Recipes in FORTRAN: The An of Scientific Computing, Cambridge Univ. press, Cambridge, MA, (1992)

[4] A. Goldsmith. T.E. Waterman and H.J. Hirschborn, Handbook of Solid Materials, The MacMillan Co., New York, USA 1961.

[5] Applications of Accelerators in Research and Industry, J.L. Duggan and I.L. Morgan (eds.), The American Institute of Physics, CP475 (1999) 929-931

[6] Radiant Properties of Materials, Aleksander Sala. PWN Polish Scientific Publishers, Warsaw, Poland (1986)

[7] Handbook of Chemistry and Physics, D.R. Lide (ed.), CRC Press, Inc. (1990)
The submitted manuscript has been created by the University of Chicago as Operator of Argonne National Laboratory ("Argonne") under Contract No. W-31-109-ENG-38 with the U.S. Department of Energy. The U.S. Government retains for itself, and others acting on its behalf, a paid-up, nonexclusive. irrevocable worldwide license in said article to reproduce, prepare derivative works, distribute copies to the public, and perform publicly and display publicly, by or on behalf of the Government. 